\title{
A DYNAMIC INVERSE FOR NONLINEAR MAPS
}

\author{
Neil H. Getz* and Jerrold E. Marsden ${ }^{\dagger}$
}

\begin{abstract}
We consider the problem of estimating the time-varying root of a time-dependent nonlinear map. We introduce a "dynamic inverse" of a map, another generally time-dependent map which one composes with the original map to form a nonlinear vector-field. The flow of this vector field decays exponentially to the root. We then show how a dynamic inverse may be determined dynamically while being used simultaneously to find a root. We construct a continuous-time analog computational paradigm around the dynamic inverse.
\end{abstract}

\section{Introduction}

In this paper ${ }^{1}$ we describe a continuous-time dynamical methodology for inverting nonlinear maps. We call this methodology dynamic inversion. Given a map $F: \mathbb{R}^{n} \times \mathbb{R}_{+} \rightarrow \mathbb{R}^{n}$ with a continuous isolated and presumably unknown solution $\theta_{*}(t)$ to $F(\theta, t)=$ 0 , we associate with $F(\theta, t)$ another map $G[w, \theta, t]$ which we call a dynamic inverse of $F(\theta, t)$. Let $E(\theta, t)$ be a smooth map such that $E\left(\theta_{*}, t\right)=\dot{\theta}_{*}$. The properties of a dynamic inverse $G[w, \theta, t]$ are such that the dynamical system

$$
\dot{\theta}=-G[F(\theta, t), \theta, t]+E(\theta, t)
$$

has a solution $\theta(t)$ which converges exponentially to the solution $\theta_{*}(t)$. This allows us to use dynamical systems of the form (1) to approximate $\theta_{*}$.

The last decade has witnessed a great deal of renewed interest in dynamic analog computational paradigms; most prominently neural networks. In another direction Brockett [2], [3] showed how one could perform operations usually accomplished with discrete algorithms, such as sorting of lists, solving of least-squares matching problems, and matrix diagonalization through the use of isospectral gradient flows on the group of orthogonal matrices. More recently Helmke and Moore [4] have studied dynamical solutions to the problems of solving for eigenvalues, singular value decomposition, and balanced matrix

* Electrical Engineering and Computer Sciences, University of California at Berkeley, Berkeley, California 94720, getz@eecs.berkeley .edu.

$\dagger$ Control and Dynamical Systems, California Institute of Technology, Pasadena, California, marsden@cds.caltech.edu.

${ }^{1}$ This paper is a condensed version of [1].

\section{0-7803-2685-7/95 \$4.00 (c) 1995 IEEE}

factorizations via gradient flows. Dynamic inversion may also be viewed as a new analog computational paradigm. Though it may be used to solve intrinsically static problems dynamically, our primary domain of application is the general class of finite dimensional inverse problems with solutions that vary in time. We make no assumption of a gradient structure in our problems, though our method may be seen to encompass gradient methods.

Nicosia, et al. [5, 6] have also considered a technique of using dynamics for nonlinear map inversion. The method we present generalizes of part of their method, though those authors also employ observers in order to estimate derivatives which, for simplicity, we will assume are known. The dynamic inverse generalizes certain maps utilized by those authors in their inversions. Also, rather than relying upon numerical matrix inversion in the inversion of nonlinear maps as done in $[5,6]$, we recognize matrix inversion as another nonlinear inversion problem, and incorporate dynamic matrix inversion into our dynamic inversions when needed.

In Section 2 we introduce the definition of a $d y$ namic inverse of a map. In Section 3 we use the dynamic inverse to construct a continuous-time dynamical estimator for time-varying vector-valued roots of nonlinear time-dependent maps. We then show how a dynamic inverse may be determined dynamically.

\section{A Dynamic Inverse}

The dynamic inverse is defined in terms of the unknown root of a map. Later we will show how a dynamic inverse may be obtained without knowing the root.

Definition 2.1 For $F: \mathbb{R}^{n} \times \mathbb{R}_{+} \rightarrow \mathbb{R}^{n} ;(\theta, t) \mapsto$ $F(\theta, t)$ let $\theta_{*}(t)$ be a continuous isolated solution of $F(\theta, t)=0 . A \operatorname{map} G: \mathbb{R}^{n} \times \mathbb{R}^{n} \times \mathbb{R}_{+} \rightarrow \mathbb{R}^{n} ;(w, t) \mapsto$ $G[w, \theta, t]$ is called a dynamic inverse of $F$ on the ball $\mathcal{B}_{r}:=\left\{z \in \mathbb{R}^{n} \mid\|z\| \leq r\right\}, r>0$, if

1. the map $G[F(\theta, t), \theta, t]$ is Lipschitz in $\theta$, continuous in $t$, and

2. there is a fixed real number $\beta$, with $0<\beta<\infty$, such that

$$
z^{T} G\left[F\left(z+\theta_{*}(t), t\right), z+\theta_{*}(t), t\right] \geq \beta\|z\|_{2}^{2}
$$


for all $z \in \mathcal{B}_{r}$.

Some easily verified properties of the dynamic inrse that prove useful are the following:

roperty 2.2 If $G[w, \theta, t]$ is a dynamic inverse of $(\theta, t)$ with parameter $\beta$, then for any $\mu>0 \in \mathbb{R}$, $\exists[w, \theta, t]$ is a dynamic inverse of $F(\theta, t)$ with pameter $\mu \beta$.

roperty 2.3 Let $\tilde{F}(z, t):=F\left(z+\theta_{*}(t), t\right)$ and let $z, \theta, t]:=G\left[w, z+\theta_{*}(t), t\right]$. Then $G[w, \theta, t]$ is a namic inverse of $F(\theta, t)$ relative to a solution $\theta_{*}$ if d only if $\tilde{G}[w, z, t]$ is a dynamic inverse of $\tilde{F}(z, t)$ lative to $z_{*}=0$.

A dynamic inverse $G$ may inherit its dependence $t$ from a variety of sources including dependence on tes of dynamical systems. The following property oves useful in this regard.

roperty 2.4 Assume that $G^{1}\left(w^{1} ; \theta^{1}, \theta^{2} ; t\right)$ is a dymic inverse of $F^{1}\left(\theta^{1}, t\right)$, for all $\theta^{2}$ satisfying $\left(\theta^{2}-\right.$ )$\in \mathcal{B}_{r_{2}}$, and $G^{2}\left(w^{2} ; \theta^{2}, \theta^{1} ; t\right)$ is a dynamic inverse $F^{2}\left(\theta^{2}, t\right)$ for all $\theta_{1}$ satisfying $\left(\theta^{1}-\theta_{*}^{1}\right) \in \mathcal{B}_{r_{1}}$. Let $:=\left(\theta^{1}, \theta^{2}\right)$ and $w=\left(w^{1}, w^{2}\right)$. Then

$$
G[w, \theta, t]:=\left[\begin{array}{c}
G^{1}\left(w^{1} ; \theta^{1}, \theta_{*}^{2} ; t\right) \\
G^{2}\left(w^{2} ; \theta^{2}, \theta_{*}^{1} ; t\right)
\end{array}\right]
$$

a dynamic inverse of

$$
F(\theta, t):=\left[\begin{array}{l}
F^{1}\left(\theta^{1}, t\right) \\
F^{2}\left(\theta^{2}, t\right)
\end{array}\right]
$$

r all $\left(\theta^{1}-\theta_{*}^{1}, \theta^{2}-\theta_{*}^{2}\right) \in \mathcal{B}_{r_{1}} \times \mathcal{B}_{r_{2}}$.

For scalar valued functions $F(\theta, t)$ we have the llowing lemma.

əmma 2.5 Let $F: \mathbb{R} \times \mathbb{R}_{+} \rightarrow \mathbb{R} ;(\theta, t) \mapsto F(\theta, t)$ be 1 in $\theta$ and continuous in $t$ for all $\theta$ is a connected terval $[a, b]$. Let $\theta_{*}(t)$ be a continuous isolated soluin of $F(\theta, t)=0$. Assume that there exists an $r>0$ Id $a \beta>0$ such that

$\left.1-\theta_{*}\right) \operatorname{sign}(F(b, t)-F(a, t)) \cdot F(\theta, t) \geq \beta\left(\theta-\theta_{*}\right)^{2}$,

$r$ all $\left(\theta-\theta_{*}\right) \in \mathcal{B}_{r}$ and all $t \in \mathbb{R}_{+}$. Then

$$
G[w]:=\operatorname{sign}\left(\frac{\partial}{\partial \theta} D_{1} F\left(\theta_{*}(0), 0\right)\right) \cdot w
$$

a constant dynamic inverse of $F(\theta, t)$.

roof: See [1] or [7].

Lemma 2.5 tells us that for time-varying scalar lued $C^{1}$ functions, we need only pick a sign to proice a dynamic inverse. Typically one knows an inrval $[a, b]$ containing the solution. One need only evaluate $F\left(a, t_{1}\right)$ and $F\left(b, t_{2}\right)$ for any times $t_{1}$ and $t_{2}$. A dynamic inverse for $F(\theta, t)$ is then $G[w]:=$ $\operatorname{sign}\left(F\left(b, t_{2}\right)-F\left(a, t_{1}\right)\right) \cdot w$.

Sufficient conditions on $F$ under which a dynamic inverse exists are mild. They are given in the following existence lemma.

Lemma 2.6 For $F: \mathbb{R}^{n} \times \mathbb{R}_{+} \rightarrow \mathbb{R}^{n} ;(\theta, t) \mapsto F(\theta, t)$, let $\theta_{*}(t)$ be a continuous isolated solution of $F(\theta, t)=$ 0 . Let $F(\theta, t)$ be $C^{2}$ in $\theta$ and continuous in $t$. Assume that the following are true:

1. $D_{1} F\left(\theta_{*}(t), t\right)$ is nonsingular for all $t$;

2. $D_{1} F\left(\theta_{*}(t), t\right)$ and $D_{1} F\left(\theta_{*}(t), t\right)^{-1}$ are bounded uniformly in $t$;

3. for all $z \in \mathcal{B}_{r}, D_{1}^{2} F\left(z+\theta_{*}(t), t\right)$ is bounded uniformly in $t$.

Under these conditions there exists an $r^{n}>0$ independent of $t$, and a function $G: \mathbb{R}^{n} \times \mathbb{R}^{n} \times \mathbb{R}_{+} \rightarrow \mathbb{R}^{n}$, $(w, \theta, t) \mapsto G[w, \theta, t]$ such that for each $t>0$ and for all $\theta$ satisfying $\theta-\theta_{*}(t) \in \mathcal{B}_{r}, G[w, \theta, t]$ is a dynamic inverse of $F(\theta, t)$.

Proof: The proof shows that an inverse function of $\theta \mapsto F(\theta, t)$ exists for each $t$ and for all $\theta$ in a ball $\mathcal{B}_{r(t)}$ about $\theta_{*}(t)$. Furthermore, for all $t \geq 0, r(t) \geq$ $r_{\min }>0$. See [7] for the complete proof.

Though Lemma 2.6 requires $F(\theta, t)$ to be $C^{2}$ in $\theta$ at $\theta=\theta_{*}(t)$, this is only a sufficient condition for the existence of a dynamic inverse as will be seen in Example 3.2 below.

One might guess that a truncated 'Taylor expansion for $F^{-1}$ might be a good candidate for $G$. That this guess is true is shown by the following example.

Example 2.7 Fixed Jacobian Inverse as a Dynamic Inverse. Let $\theta_{*}(t)$ be a continuous isolated solution of $F(\theta, t)=0$, with $\theta \in \mathbb{R}^{n}$ and $t \in \mathbb{R}_{+}$. Let $t_{1} \in \mathbb{R}_{+}$be fixed with $t_{1}>0$. Let $\tilde{F}(z, t):=$ $F\left(z+\theta_{*}(t), t\right), z \in \mathbb{R}_{+}$, have the Taylor series expansion in both variables

$$
\tilde{F}(z, t)=D_{1} \tilde{F}\left(0, t_{1}\right) \cdot z+O\left(\|z\|^{2},\left|t-t_{1}\right|\|z\|\right)
$$

where the form of the error uses the fact that $\tilde{F}(0, t)=$ 0 for all $t$, and as a consequence $D_{2}^{k} \tilde{F}\left(0, t_{1}\right) \equiv 0$ for $k \in\{1,2, \ldots\}$. Choose $r>0$ and expand $D_{1} \tilde{F}\left(0, t_{1}\right)$ about some $y \in \mathcal{B}_{r} \subset \mathbb{R}^{n}$ as

$$
D_{1} \tilde{F}\left(0, t_{1}\right)=D_{1} \tilde{F}\left(y, t_{1}\right)+O(\|y\|) .
$$

Note that $y$ need not be constant, or even continuous in time. Substituting (8) into (7) gives

$$
\tilde{F}(z, t)=D_{1} \tilde{F}\left(y, t_{1}\right) \cdot z+f(z, t)
$$


where

$$
f(z, t)=O\left(\|z\|^{2},\left|t-t_{1}\right|\|z\|,\|y\|\|z\|\right) .
$$

Now consider the dynamic inverse candidate

$$
G[w, \theta, t]=G[w]=D_{1} \tilde{F}\left(y, t_{1}\right)^{-1} \cdot w .
$$

Substitute $G$ and $\tilde{F}$ into the dynamic inverse criterion (2), and expand $\tilde{F}$ according to (9) to get

$$
\begin{aligned}
z^{T} G[\tilde{F}(z, t)] & =z^{T} D_{1} \tilde{F}\left(y, t_{1}\right)^{-1} \tilde{F}(z, t) \\
& =z^{T} z+z^{T} D_{1} \tilde{F}\left(y, t_{1}\right)^{-1} f\left(z, t_{1} \cdot 2\right)
\end{aligned}
$$

Let $\beta \in \mathbb{R}_{+}$be some fixed number with $1>\beta>$ 0 . If there exists an $r \in \mathbb{R}_{+}$and an interval $\left(t_{0}, t_{2}\right)$ containing $t_{1}$ such that for all $z \in \mathcal{B}_{r}$ and all $t \in$ $\left(t_{0}, t_{2}\right)$,

$$
z^{T} D_{1} \tilde{F}\left(y, t_{1}\right)^{-1} f(z, t) \geq(\beta-1)\|z\|_{2}^{2},
$$

then $G$ is a dynamic inverse of $\tilde{F}$ on $\mathcal{B}_{r}$ as long as $t$ is in $\left(t_{0}, t_{2}\right)$. Since $f(z, t)$ satisfies $(10)$, it is also true that

$D_{1} \tilde{F}\left(y, t_{1}\right)^{-1} \cdot f(z, t)=O\left(\|z\|^{2},\left|t-t_{1}\right|\|z\|,\|y\|\|z\|\right)$.

Thus there are always sufficiently small $r>0$, and a sufficiently small interval $\left(t_{0}, t_{2}\right)$ such that $(13)$ is true.

Remark 2.8 Nearby Jacobian Inverse as a Dynamic Inverse. We may replace $t_{1}$ by $t$ in Example 2.7 to conclude that $D_{1} \tilde{F}(\theta(t), t)^{-1}$ is a dynamic inverse of $F(\theta, t)$ for all $t$ if $\theta(t)$ is sufficiently close to $\theta_{*}(t)$ for all $t \in \mathbb{R}_{+}$.

Remark 2.9 If $F(\theta, t)$ is $C^{1}$ in $\theta$, then any $B(\theta, t) \in$ $\mathbb{R}^{n \times n}$ such that $B$ is continuous in $t$, and

$$
B\left(\theta_{*}, t\right) D_{1} F\left(\theta_{*}, t\right)>0
$$

is a dynamic inverse for $F$. This includes as special cases $B(t)=D_{1} F\left(\theta_{*}, t\right)^{-1}$ and $B(t)=D_{1} F\left(\theta_{*}, t\right)^{T}$, as well as $B(t)=D_{1} F(\theta, t)^{-1}$ and $B(t)=D_{1} F(\theta, t)^{T}$ where $\left\|\theta-\theta_{*}\right\|$ is sufficiently small.

Nonlinear Dynamic Inverses. Though it will often be convenient to choose a linear dynamic inverse, a dynamic inverse need not be linear as shown by the following two examples.

Example 2.10 Let $F(\theta, t)=(\theta-\sin (t))^{3}$. Then $\theta_{*}=\sin (t)$. Note that $F(\theta, t)$ fails to satisfy the conditions of Lemma 2.6. Let $G[w]:=\operatorname{sign}(w)|w|^{1 / 3}$ Then

$$
z^{T} G\left[F\left(z+\theta_{*}, t\right)\right]=z^{T} z \geq\|z\|^{2}
$$

so $G[w]$ is a dynamic inverse of $F(\theta, t)$.
Example 2.11 Let

$$
F(\theta, t)=\tan (\theta-\sin (t))
$$

We may obtain a dynamic inverse of $F$ through Taylor series reversion ${ }^{2}$ of $\tan (z)$. Let $G[w, \theta, t]=G[w]=$ $w-w^{3} / 3$. It is easily verified graphically that $G[w]$ is a dynamic inverse of $F(\theta, t)$ for $\beta=1 / 4$ and for all $z \in \mathcal{B}_{1}$.

Later in section 4 we will show how a dynamic inverse can be determined dynamically, that is, we will find both the root and the dynamic inverse itself using the same dynamical system.

\section{Dynamic Inversion}

In this section we will use the dynamic inverse to construct a dynamical system whose state is an estimator for the root $\theta_{*}(t)$ of $F(\theta, t)=0$. We give a dynamic inversion theorem which guarantees exponential convergence of an approximation error to zero.

It is often the case that $F(\theta, t)$ is differentiable in $\theta$ and $t$. Under this condition an estimator, $E(\theta, t)$, for $\dot{\theta}_{*}$ can be obtained by differentiating $F\left(\theta_{*}(t), t\right)=0$ with respect to $t$, solving for $\dot{\theta}_{*}$, and substituting $\theta$ for $\theta_{*}$. In general such an estimator will depend on $t$ as well as an estimate of $\theta_{*}$. The estimator may become arbitrarily precise as the estimate of $\theta_{*}(t)$ approaches $\theta_{*}(t)$. Approximations of $E(\theta, t)$, such as those based upon a truncated Taylor series expansion of $E(\theta, t)$, may also possess this property. Intuitively one might hope to produce an estimator for $\dot{\theta}_{*}(t)$ which, even though it depends upon an estimator of $\theta_{*}$, leads to an approximator $\theta(t)$ that converges exponentially to $\theta_{*}(t)$. We will formalize that intuition in Theorem 3.1 .

Theorem 3.1 Let $\theta_{*}(t)$ be a continuous isolated solution of $F(\theta, t)=0$, with $F: \mathbb{R}^{n} \times \mathbb{R}_{+} \rightarrow \mathbb{R}^{n}$; $(\theta, t) \mapsto F(\theta, t)$. Assume that $G: \mathbb{R}^{n} \times \mathbb{R}^{n} \times \mathbb{R}_{+} \rightarrow \mathbb{R}^{n} ;$ $(w, \theta, t) \mapsto G[w, \theta, t]$, is a dynamic inverse of $F(\theta, t)$ on $\mathcal{B}_{r}$, for some finite $\beta>0$. Let $E: \mathbb{R}^{n} \times \mathbb{R}_{+} \rightarrow \mathbb{R}^{n}$; $(\theta, t) \mapsto E(\theta, t)$ be locally Lipschitz in $\theta$ and continuous in $t$. Assume that for some fixed $\kappa \in(0, \infty)$, $E(\theta, t)$ satisfies

$$
\left\|E\left(z+\theta_{*}(t), t\right)-\dot{\theta}_{*}(t)\right\|_{2} \leq \kappa\|z\|_{2}
$$

for all $z \in \mathcal{B}_{r}$. Let $\theta(t)$ denote the solution to the system

$$
\dot{\theta}=-\mu G(F(\theta, t), \theta, t)+E(\theta, t)
$$

\footnotetext{
${ }^{2}$ One reverts a Taylor series, $q(z)=a_{0}+a_{1} z+a_{2} z^{2}+O\left(|\theta|^{3}\right)$ by solving for the coefficients $b_{i}$ of a polynomial $p(z)=b_{0}+$ $b_{1}(z)+b_{2}(z)^{2}$ under the constraint that $p(q(z))=1$.
} 
with initial condition $\theta(0)$ satisfying $\theta(0)-\theta_{*}(0) \in \mathcal{B}_{r}$. Then

$$
\left\|\theta(t)-\theta_{*}(t)\right\|_{2} \leq\left\|\theta(0)-\theta_{*}(0)\right\|_{2} e^{-(\mu \beta-\kappa) t}
$$

for all $t \in \mathbb{R}_{+}$, and in particular if $\mu>\kappa / \beta$, then $\theta(t)$ converges to $\theta_{*}(t)$ exponentially.

Proof: Let $z(t):=\theta(t)-\theta_{*}(t), \tilde{F}(z, t):=F(z+$ $\left.\theta_{*}(t), t\right)$, and $\tilde{G}[w, z, t]:=G\left[w, z+\theta_{*}(t), t\right]$. Differentiate $z(t)=\theta(t)-\theta_{*}(t)$ with respect to $t$, and use (18) to get

$$
\dot{z}=-\mu \tilde{G}(\tilde{F}(z, t), z, t)+E\left(z+\theta_{*}(t), t\right)-\dot{\theta}_{*}(t) .
$$

Let

$$
V(z):=\frac{1}{2}\|z\|_{2}^{2} .
$$

Differentiate $V$ with respect to $t$ to get

$$
\begin{aligned}
\frac{d}{d t} V(z)= & -\mu z^{T} \tilde{G}(\tilde{F}(z, t), z, t) \\
& +z^{T}\left(E\left(z+\theta_{*}(t), t\right)-\dot{\theta}_{*}(t)\right) .
\end{aligned}
$$

Then by Definition 2.1 and (17),

$$
\frac{d}{d t} V(z) \leq-\mu \beta\|z\|_{2}^{2}+k\|z\|_{2}^{2}=-(\mu \beta-k)\|z\|_{2}^{2}
$$

so that for $z \in \mathcal{B}_{r}$ we have

$$
\frac{d}{d t} V(z) \leq-2(\mu \beta-k) V(z) .
$$

Thus

$$
V(z) \leq \frac{1}{2}\|z(0)\|_{2}^{2} e^{-2(\mu \beta-k) t}
$$

and consequently

$$
\|z(t)\| \leq\|z(0)\|_{2} e^{-(\mu \beta-k) t} .
$$

Example 3.2 Consider the piecewise-linear map $F$ : $[-4,4] \subset \mathbb{R} \rightarrow \mathbb{R}$ defined by

$$
F(\theta, t)=\sin (4 \pi t)+\left\{\begin{array}{cl}
-4-\theta / 2, & \theta<-2 \\
3 \theta / 2, & -2 \leq \theta \leq 2 . \\
4-\theta / 2, & \theta>2
\end{array}\right. \text { (27) }
$$

The unique solution of $F(\theta, t)=0$ in $(-4,4)$ is $\theta_{*}(t)=$ $-(2 / 3) \sin (4 \pi t)$, and we may differentiate $\theta_{*}(t)$ to obtain $\dot{\theta}_{*}(t)=-(8 \pi / 3) \cos (4 \pi t)$. A dynamic inverse of $F(\theta, t)$ is $G[w, \theta, t]=w$ corresponding to $\beta=1$. Let

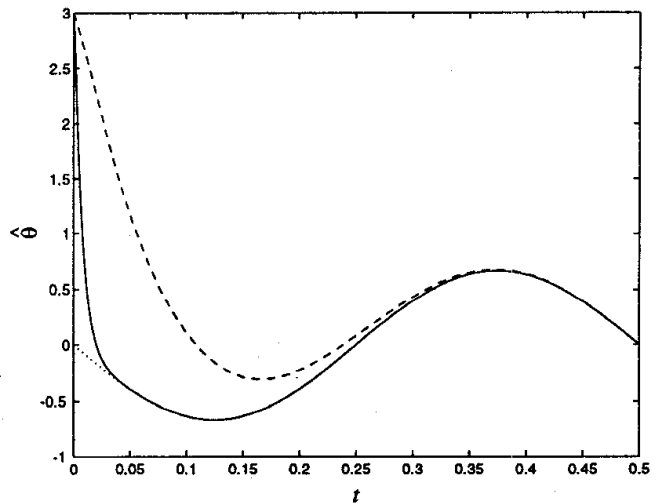

Figure 1: Solutions of the dynamic inverter of Example 3.2 with $E(\theta, t)=\dot{\theta}_{*}(t)$ for $\mu=10$ (dashed), $\mu=100$ (solid), and the actual solution $\theta_{*}(t)$ (dotted). The initial condition was $\theta(0)=3$.

$E(\theta, t):=-(8 \pi / 3) \cos (4 \pi t)$ and use the dynamic inverter

$$
\dot{\theta}=-\mu F(\theta, t)+E(\theta, t)
$$

with the same initial condition as before, $\theta(0)=3$. Figure 1 shows the simulation results. In this case the errors can be seen to go to zero exponentially.

Note that for (28), each of the two solutions $\theta(t)$, corresponding to each of the two values of $\mu$ in the simulations, pass through the point $\theta=2$, a local maximum of $F$, for which $F(\theta(t), t)$ is not differentiable. In contrast, Newton's method and the gradient method are undefined for non-differentiable functions, and even if we were to make $F(\theta, t)$ differentiable in $\theta$ by smoothing it, Newton's method would fail due to the local maximum at $\theta=2$.

Remark 3.3 If $\theta(0)=\theta_{*}(0)$, then the conditions of Theorem 3.1 guarantee that $\theta(t)=\theta_{*}(t)$ for all $t \in$ $\mathbb{R}_{+}$. So in a sense, we need only solve the inverse problem at a single instant $t=0$. Then the dynamic inversion takes care of maintaining the inversion for all $t$.

Remark 3.4 Let

$$
G[w, \theta, t]:=D_{1} F(\theta, t)^{-1} \cdot w .
$$

It follows from Lemma 2.6 and Theorem 3.1 that if $\mu$ is sufficiently large, $\left\|\theta(0)-\theta_{*}(0)\right\|$ is sufficiently small, and $G[w, \theta(0), 0]$ is a dynamic inverse of $F(\theta, t)$ at $t=0$, then $G[w, \theta, t]$ is a dynamic inverse of $F(\theta, t)$ for all $t>0$.

Example 3.5 will illustrate application of Remark 3.4 to the estimation of $\theta_{*}(t)$. 
Example 3.5 Let $w$ and $\theta$ be in $\mathbb{R}^{n}$. Assume that the assumptions of Lemma 2.6 hold. We may obtain an estimator $E(\theta, t)$ for $\dot{\theta}_{*}$ by differentiating $F\left(\theta_{*}(t), t\right)=$ 0 ,

$$
D_{1} F\left(\theta_{*}(t), t\right) \dot{\theta}_{*}(t)+D_{2} F\left(\theta_{*}, t\right)=0,
$$

solving for $\dot{\theta}_{*}$, and replacing $\theta_{*}$ by $\theta$ to get

$$
E(\theta, t):=-D_{1} F(\theta, t)^{-1} D_{2} F(\theta, t) .
$$

Assume that $r$ has been chosen sufficiently small, and that $D_{2} F(\theta, t)$ is sufficiently bounded so that $E(\theta, t)$ satisfies (17) for all $z \in \mathcal{B}_{r}$. Let

$$
G[w, \theta, t]:=D_{1} F(\theta, t)^{-1} \cdot w
$$

and assume that $r$ is small enough that $G$ is a dynamic inverse of $F$ on $\mathcal{B}_{r}$. If $\left(\theta(0)-\theta_{*}(0)\right) \in \mathcal{B}_{r}$, and $\mu$ is sufficiently large, then by Theorem 3.1 the approximation error $z(t):=\theta(t)-\theta_{*}(t)$ using (18) will converge exponentially to zero.

\section{Dynamic Estimation of a Dynamic Inverse}

In this section we will show how we can apply the dynamic inversion theorem to the construction of a dynamical system whose state includes both a dynamic inverse of a particular $F$ as well as an approximation for the root of $F$. Consideration of the example of dynamic inversion of a time-varying matrix [8] will lead the way.

Example 4.1 Consider the problem of estimating the inverse $\Gamma_{*}(t) \in \mathbb{R}^{n \times n}$ of a time-varying matrix $A(t) \in G L(n, \mathbb{R})$, where $G L(n, \mathbb{R})$ denotes the group of invertible matrices in $\mathbb{R}^{n \times n}$. Assume that we have representations for both $A(t)$ and $\dot{A}(t)$, and that $A(t)$ is $C^{1}$ in $t$. Let $\Gamma$ be an element of $\mathbb{R}^{n \times n}$.

In order for $\Gamma_{*}$ to be the inverse of $A(t), \Gamma_{*}$ must satisfy

$$
A(t) \Gamma-I=0 .
$$

Let $F^{\gamma}: \mathbb{R}^{n \times n} \times \mathbb{R}_{+} \rightarrow \mathbb{R}^{n \times n} ;(\Gamma, t) \mapsto F^{\gamma}(\Gamma, t)$ be defined by

$$
F^{\gamma}(\Gamma, t):=A(t) \Gamma-I .
$$

Let the solution of $F^{\gamma}(\Gamma, t)=0$ be $\Gamma_{*}(t)$. We obtain an estimator $E^{\gamma}(\Gamma, t)$ for $\dot{\Gamma}_{*}(t)$ by differentiating $A \Gamma_{*}=I$ with respect to $t$, solving the resulting expression for $\dot{I}_{*}$, replacing $A^{-1}$ by $\theta_{*}$, and then replacing $\Gamma_{*}$ by $\Gamma$ in the resulting expression to get

$$
E^{\gamma}(\Gamma, t):=-\Gamma \dot{A}(t) \Gamma .
$$

Differentiate $F^{\gamma}(\Gamma, t)$ with respect to $\Gamma$ to get

$$
D_{1} F^{\gamma}(\Gamma, t)=A(t)
$$

whose inverse is $\Gamma_{*}$. So a choice of dynamic inverse is

$$
\tilde{G}^{\gamma}[w, \Gamma, t]:=\Gamma \cdot w
$$

for $\Gamma$ sufficiently close to $\Gamma_{*}=A^{-1}(t)$. The dynamic inverter for this problem then takes the form

$$
\begin{aligned}
\dot{\Gamma} & =-\mu G^{\gamma}\left[F^{\gamma}(\Gamma, t), \Gamma\right]+E^{\gamma}(\Gamma, t) \\
& =-\mu \Gamma(A(t) \Gamma-I)-\Gamma \dot{A}(t) \Gamma .
\end{aligned}
$$

and we choose as initial conditions $\Gamma(0) \approx \Gamma_{*}(0)=$ $A^{-1}(0)$ so that the estimation error starts small. Theorem 3.1 guarantees that for sufficiently large $\mu$, and for $\Gamma(0)$ sufficiently close to $A^{-1}(0)$, equation (38) will produce an estimator $\Gamma$ whose error decays exponentially to zero at a rate determined by our choice of $\mu$.

Remark 4.2 Example 4.1 also allows one to invert time-varying matrices without calling upon discrete matrix inversion routines. One only need calculate or approximate a single inverse, $A(0)^{-1}$. The flow of (38) then maintains the inversion for all $t>0$.

Example 4.3 Assume that $F(\theta, t)$ satisfies the assumptions of Lemma 2.6 with continuous isolated solution $\theta_{*}$. Assume that $D_{1} F(\theta, t)$ is $C^{2}$ in $\theta$ and $C^{1}$ in $t$. Let $\Gamma \in \mathbb{R}^{n \times n}$ denote an estimator for $D_{1} F\left(\theta_{*}, t\right)^{-1}$. We may then estimate $\dot{\theta}_{*}(t)$ as follows: Differentiate $F\left(\theta_{*}, t\right)=0$, solve for $\dot{\theta}_{*}$, and substitute $\Gamma$ for $D_{1} F\left(\theta_{*}(t), t\right)^{-1}$ and $\theta$ for $\theta_{*}$ to obtain an estimator for $\dot{\theta}_{*}$ in terms of $\Gamma, \theta$, and $t$,

$$
E(\Gamma, \theta, t):=-\Gamma D_{2} F(\theta, t) .
$$

Assume that $E(\Gamma, \theta, t)$ is $C^{1}$ in its arguments. Using $E(\Gamma, \theta, t)=\left[E_{i}(\Gamma, \theta, t)\right]_{i \in n}$, by (35) we may estimate $\dot{\Gamma}_{*}$ with

$$
E^{\gamma}(\Gamma, \theta, t):=-\left.\Gamma \frac{d}{d t} D_{1} F(\theta, t)\right|_{\dot{\theta}=E(\Gamma, \theta, t)} \Gamma
$$

where

$$
\begin{aligned}
& \left.\frac{d}{d t} D_{1} F(\theta, t)\right|_{\dot{\theta}=E(\Gamma, \theta, t)}:= \\
& \sum_{i=1}^{n} \frac{\partial D_{1} F(\theta, t)}{\partial \theta_{i}} E_{i}(\Gamma, \theta, t)+\frac{\partial D_{1} F(\theta, t)}{\partial t} .
\end{aligned}
$$

In this case

$$
F^{\gamma}(\Gamma, \theta, t):=D_{1} F(\theta, t) \Gamma-I .
$$

Let $G \gamma[w, \Gamma]:=\Gamma \cdot w$ as in Example 4.1.Theorem 3.1 now tells us that we may estimate $\theta_{*}(t)$ with the system of coupled nonlinear differential equations

$$
\begin{aligned}
{\left[\begin{array}{c}
\dot{\Gamma} \\
\dot{\theta}
\end{array}\right]=} & -\mu\left[\begin{array}{cc}
\Gamma & 0 \\
0 & \Gamma
\end{array}\right] \cdot\left[\begin{array}{c}
F^{\gamma}(\Gamma, \theta, t) \\
F(\theta, t)
\end{array}\right] \\
& +\left[\begin{array}{c}
E^{\gamma}(\Gamma, \theta, t) \\
E(\Gamma, \theta, t)
\end{array}\right]
\end{aligned}
$$


with guaranteed exponential convergence of $(\Gamma, \theta)$ to $\left(\Gamma_{*}, \theta_{*}\right)$.

After a definition, we summarize the result of Example 4.3 with the following theorem.

Definition 4.4 For $(\Gamma, \theta) \in \mathbb{R}^{n \times n} \times \mathbb{R}^{n}$, let

$$
\|(\Gamma, \theta)\|_{F}:=\left(\sum_{i, j=1}^{n}\left|\Gamma_{i j}\right|^{2}+\sum_{i=1}^{n} \theta_{i}\right)^{1 / 2} .
$$

This is equivalent to the Froebenius norm ${ }^{3}$ of the ma$\operatorname{trix}[\Gamma, \theta]$. Let the corresponding open ball in this norm be denoted $\mathcal{B}_{r}^{F}$.

Theorem 4.5 Let $F(\theta, t)$ satisfy the assumptions of Lemma 2.6. For $\Gamma(0)$ sufficiently close to $D_{1} F\left(\theta_{*}, 0\right)^{-1}$, and $\theta(0)$ sufficiently close to $\theta_{*}(0)$, the solution $(\Gamma(t), \theta(t))$ of

$$
\begin{aligned}
& {\left[\begin{array}{c}
\dot{\Gamma} \\
\dot{\theta}
\end{array}\right]=-\mu\left[\begin{array}{cc}
\Gamma & 0 \\
0 & \Gamma
\end{array}\right]\left[\begin{array}{c}
D_{1} F(\theta, t) \Gamma-I \\
F(\theta, t)
\end{array}\right]} \\
& +\left[\begin{array}{c}
-\left.\Gamma \frac{d}{d t} D_{1} F(\theta, t)\right|_{\dot{\theta}=E(\Gamma, \theta, t)} \Gamma \\
-\Gamma D_{2} F(\theta, t)
\end{array}\right],
\end{aligned}
$$

where $E(\Gamma, \theta, t)$ is given by (39), satisfies $(\Gamma(t), \theta(t)) \rightarrow$ $\left(D_{1} F\left(\theta_{*}, t\right), \theta_{*}(t)\right)$ as $t \rightarrow \infty$. Furthermore, for sufficiently large $\mu>0$, the convergence is exponential, i.e. there exists a $k>0$ such that

$$
\begin{aligned}
& \|(\Gamma(t), \theta(t))-\left(\Gamma_{*}(t), \theta_{*}(t) \|_{F}\right. \\
& \leq \|(\Gamma(0), \theta(0))-\left(\Gamma_{*}(0), \theta_{*}(0) \|_{F} e^{-k t}\right.
\end{aligned}
$$

for all $t \geq 0$.

\section{Summary}

A methodology for constructing dynamical systems for tracking roots of nonlinear time-dependent maps has been presented and shown to have exponentially decaying error.

For examples of application of Theorem 4.5 to tracking control of a simple robot arm, see [10,7]. For an example of the application of Theorem 4.5 to matrix inversion and polar decomposition see $[11,8,7]$. For an example of the application of Theorem 4.5 to the problem of tracking implicitly defined trajectories, see $[12,7]$.

The continuous dynamical approach of dynamic inversion has the virtue that it is independent of implementation. It may be realized in an analog as well as a digital manner (through association with an integrator). In the control context, dynamic inversion in combination with a continuous-time plant and controller allows a seamless incorporation of rootsolving without the need to mix continuous-time and discrete-time analysis.

\footnotetext{
${ }^{3}$ Also called the Schur norm or Hilbert-Schmidt norm. See Horn and Johnson [9].
}

Acknowledgements. The authors are grateful to C.A. Desoer for his comments and advice.

\section{References}

[1] N. H. Getz and J. E. Marsden, "Dynamic inversion of nonlinear maps," Tech. Rep. 621, Center for Pure and Applied Mathematics, Berkeley, California, 19 December 1994.

[2] R. W. Brockett, "Dynamical systems that sort lists, diagonalize matrices, and solve linear programming problems," Linear Algebra and Its Applications, vol. 146, pp. 79-91, February 1991.

[3] R. W. Brockett, "Least squares matching problems," Linear Algebra and Its Applications, vol. 122, pp. 761-777, Sep-Nov 1989.

[4] U. Helmke and J. B. Moore, Optimization and Dynamical Systems. Communications and Control Engineering, New York: Springer-Verlag, 1994.

[5] S. Nicosia, A. Tornambè, and P. Valigi, "A solution to the generalized problem of nonlinear map inversion," Systems and Control Letters, vol. 17, no. 5, 1991.

[6] S. Nicosia, A. Tornambè, and P. Valigi, "Use of observers for the inversion of nonlinear maps," Systems and Control Letters, vol. 16, no. 6, 1991.

[7] N. H. Getz, Dynamic Inversion of Nonlinear Maps with Applications to Nonlinear Control. $\mathrm{PhD}$ thesis, University of Califormia at Berkeley, 1995.

[8] N. H. Getz and J. E. Marsden, "Dynamical methods for polar decomposition and inversion of matrices," Tech. Rep. 624, Center for Pure and Applied Mathematics, Berkeley, California, 5 January 1995. Submitted to Linear Algebra and its Applications.

[9] R. A. Horn and C. R. Johnson, Matrix Analysis. New York: Cambridge University Press, 1985.

[10] N. H. Getz and J. E. Marsden, "Joint space tracking of workspace trajectories in continuous time," in 34th IEEE Conference on Decision and Control, (New Orleans), IEEE, 13-15 December 1995.

[11] N. H. Getz and J. E. Marsden, "Dynamic inversion and polar decompostion of matrices," in 34th IEEE Conference on Decision and Control, (New Orleans), IEEE, 13-15 December 1995.

[12] N. H. Getz and J. E. Marsden, "Tracking implicit trajectories," in IFAC Symposium on Nonlinear Control Systems Design, (Tahoe City), International Federation of Automatic Control, June 1995. 